\title{
Naturally large radiative lepton flavor violating Higgs decay mediated by lepton-flavored dark matter
}

\author{
Seungwon Baek and Zhaofeng Kang \\ School of Physics, KIAS, \\ 85 Hoegiro Dongdaemun-gu, Seoul 02455, Korea \\ E-mail: swbaek@kias.re.kr, zhaofengkang@gmail.com
}

\begin{abstract}
In the standard model (SM), lepton flavor violating (LFV) Higgs decay is absent at renormalizable level and thus it is a good probe to new physics. In this article we study a type of new physics that could lead to large LFV Higgs decay, i.e., a lepton-flavored dark matter (DM) model which is specified by a Majorana DM and scalar lepton mediators. Different from other similar models with similar setup, we introduce both left-handed and right-handed scalar leptons. They allow large LFV Higgs decay and thus may explain the tentative $\operatorname{Br}(h \rightarrow \tau \mu) \sim 1 \%$ experimental results from the LHC. In particular, we find that the stringent bound from $\tau \rightarrow \mu \gamma$ can be naturally evaded. One reason, among others, is a large chirality violation in the mediator sector. Aspects of relic density and especially radiative direct detection of the leptonic DM are also investigated, stressing the difference from previous lepton-flavored DM models.
\end{abstract}

Keywords: Beyond Standard Model, Higgs Physics

ARXIV EPRINT: 1510.00100 


\section{Contents}

1 Introduction and motivation 1

2 Lepton-flavored Majorana dark matter 3

2.1 The model with dual mediators 3

2.2 The mass spectrum of the mediators 4

$3 \quad h \rightarrow \tau \mu$ confronting $\tau \rightarrow \mu \gamma$ and $h \rightarrow \gamma \gamma$

3.1 Radiative LFV Higgs decay: $h \rightarrow \bar{\ell}_{a} \ell_{b}$

3.2 Induced CLFV $\tau \rightarrow \mu \gamma \quad 7$

3.3 Hints in $h \rightarrow \gamma \gamma \quad 9$

$\begin{array}{ll}3.4 \text { Natural ways to get large } h \rightarrow \tau \mu & 10\end{array}$

4 Leptophilic DM: relic density \& direct detection $\quad 12$

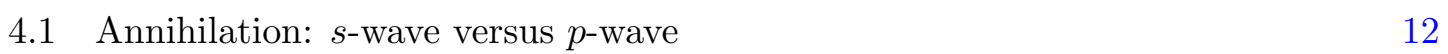

4.2 On (in)direct searches for the leptophilic DM 13

$\begin{array}{lll}5 & \text { Conclusion } & 15\end{array}$

A Two- and three-points scalar functions and their limits 16

$\begin{array}{ll}\text { B Radiative corrections on Higgs-DM-DM vertex } & 18\end{array}$

\section{Introduction and motivation}

After the discovery of standard model (SM) Higgs-like boson, the next step is to measure its couplings precisely to see possible deviation from the SM and thus to search for new physics. The Yukawa couplings between Higgs boson and charged leptons that cause lepton flavor violation (LFV) are of particular interest, because in the SM they are absent at tree level and highly suppressed at loop levels, and thus are sensitive to new physics. As a matter of fact, in most of models that address neutrino masses and oscillations, LFV is well expected and has already been observed in neutrino oscillations described by the PMNS matrix. Moreover, although charged lepton flavor violation (CLFV) has not been observed yet, in general those models should leave measurable signals in processes like $\mu \rightarrow e \gamma, \mu \rightarrow 3 e$, etc. A lot of efforts have been devoted to searching for CLFV and the null results impose very strong bounds on the magnitude of LFV [1].

Searching for LFV Higgs decays [2] receives special attention in the LHC era [3]. The CMS collaboration reported the upper limit $\operatorname{Br}(h \rightarrow \tau \mu)<1.57 \%$ at 95\% C.L., using the $19.7 \mathrm{fb}^{-1}$ of $\sqrt{s}=8 \mathrm{TeV}$ data [4]. Interestingly, the best fit (assuming both the production cross section and total width of Higgs being SM-like) hints a $2.4 \sigma$ excess with 
$\operatorname{Br}(h \rightarrow \tau \mu)=\left(0.84_{-0.37}^{+0.39}\right) \%$. More recently, the ATLAS collaboration obtained an the upper limit $\operatorname{Br}(h \rightarrow \tau \mu)<1.85 \%$ from hadronic $\tau$ decay at $95 \%$ C.L., using the $20.3 \mathrm{fb}^{-1}$ of $\sqrt{s}=8 \mathrm{TeV}$ data [5]. Although they have not seen significant deviation from the SM, their best fit value $\operatorname{Br}(h \rightarrow \tau \mu)=(0.77 \pm 0.62) \%$ is consistent with the CMS result. At the $300 \mathrm{fb}^{-1}$ of $13 \mathrm{TeV}$ LHC, the sensitivity can reach down to $7.7 \times 10^{-4}$ and thus the CMS excess will be confirmed or excluded [6].

In the models with canonical seesaw mechanism LFV Higgs decay is too small to be observed $[7,8]$. This is because of the decoupling of right-handed neutrinos (RHNs) either through the smallness of Yukawa couplings or heaviness of RHNs. In the inverse seesaw mechanism, where sizable Yukawa couplings are allowed for light RHNs, appreciable LFV Higgs decay can be accommodated $[9,10]$.

Alternatively, the tiny neutrino masses can be generated by radiative corrections [1114]. However, to our knowledge, none of those radiative seesaw models could generate large LFV Higgs decay. Actually, facing the stringent constraint from CLFV, it is quite nontrivial to get LFV Higgs decay large enough to detect at the LHC.

At tree level, two (or even more)-Higgs doublet model (2HDM) with proper flavor changing neutral current allows LFV Higgs decay which is large enough to explain the CMS excess [15-25]. Higher dimensional operators in the effective theory framework were also considered [19, 23, 28, 29]. But at loop level a large cancellation probably is needed to evade the CLFV constraint [19, 30, 31]. Other scenarios can be found in ref. [26, 27].

In this article we establish a connection between LFV Higgs decay and a type of dark matter (DM), i.e., lepton-flavored DM [32-40]. In this scenario, DM interacts merely with the SM lepton sector, whereupon DM-quark interactions arise at loop level. An obvious merit of that kind of DM is that we can easily understand the null results from DM direct detection experiments such as LUX [41].

That paradigm can be achieved in two ways. One way is introducing a leptophilic vector boson or Higgs boson propagating in the $s$-channel for the DM pair annihilation diagrams. This kind of model gives rise to poor flavor phenomenology.

The other way is introducing mediators in the $t$-channel to form lepton flavored DM. ${ }^{1}$ Then, LFV can happen in the dark sector and is mediated to the SM sector via loop processes. Furthermore, mediators could consist of both left-handed and right-handed scalar leptons (the previous studies were based on only one type of them), just as in the case of the supersymmetric SMs. Remarkably, we find that this kind of lepton-flavored DM is able to accommodate LFV Higgs decay while other models with only one type chirality fail to. As an example, we will show that in our model a sizable $\operatorname{Br}(h \rightarrow \tau \mu)$ at the level of $1 \%$ can be naturally achieved without incurring too large $\operatorname{Br}(\tau \rightarrow \mu \gamma)$. It is attributed partly to the large chirality flipping in the scalar sector and also to the cancellation between different contributions to CLFV. In addition, we study the mechanism for DM, a Majorana fermion, to acquire correct relic density. For the weak scale DM, even $s$-wave annihilation may work without large Yukawa couplings. Related to radiative LFV Higgs decay, radiative

\footnotetext{
${ }^{1}$ Note that in this way the leptonic nature of DM is naturally specified by the quantum numbers of mediators with respect to SM. No extra local or global leptonic symmetry is required.
} 
correction could also lead to Higgs-mediated DM-nucleon scattering which may be detected in the near future.

This paper is organized as follows: in section 2 the model is introduced. In section 3 we consider Higgs LFV decay confronting charged lepton LFV decay, along with others. In section 4 we study relic density and direct detection of our leptophilic dark matter and their relations with LFV Higgs decay. We conclude in section 5.

\section{Lepton-flavored Majorana dark matter}

In this section we will first present the model in its simplest version, and then calculate the mass spectra that will be used later.

\subsection{The model with dual mediators}

From model building perspective, a natural way to realize a lepton-flavored DM is to introduce a Majorana DM candidate connected to some lepton flavors by means of scalar leptons. If DM is a scalar field, whether it is real or complex, it is hard to get rid of the conventional Higgs portal in a natural manner, not to mention other demerits. So we focus on the case where DM is a singlet Majorana fermion $N$, protected by a $Z_{2}$ dark matter parity. At the renormalizable level, $N$ can not couple to SM fields. Its interactions with SM fields necessitate additional mediators, and we can specify these interactions by introducing mediators with proper quantum numbers. In order to make up a lepton-flavored DM, one can designate a scalar partner for each SM left-handed lepton doublet $l_{L}$ and right-handed lepton signlet $e_{R}$. They are labelled as $\phi_{\ell}$ and $\phi_{e}$, respectively. For simplicity, only a single family of scalar lepton (slepton for short, borrowing the name from supersymmetry) will be considered. In this paper we do not have the ambition to address the flavor structure of the dark sector by imposing flavor symmetry. We just treat all the couplings as free parameters.

With the degrees of freedom at hand, restricted by the $Z_{2}$ dark matter parity under which only the new particles are odd, the most general Lagrangian (aside from the kinetic energy terms) takes a form of

$$
\begin{aligned}
-\mathcal{L}= & -\mathcal{L}_{\mathrm{SM}}+m_{\phi_{l}}^{2}\left|\phi_{\ell}\right|^{2}+m_{\phi_{e}}^{2}\left|\phi_{e}\right|^{2}+\frac{1}{2} M \bar{N} N+\left(-y_{L a} \bar{l}_{a} P_{R} N \widetilde{\phi}_{\ell}+y_{R a} \bar{e}_{a} P_{L} N \phi_{e}+\text { h.c. }\right) \\
& +\left(-\mu H^{\dagger} \widetilde{\phi}_{\ell} \phi_{e}^{*}+\text { h.c. }\right)+\lambda_{-1}\left|\phi_{e}\right|^{2}\left|\phi_{\ell}\right|^{2}+\lambda_{0}|H|^{2}\left|\phi_{e}\right|^{2}+V_{2 \mathrm{HDM}},
\end{aligned}
$$

where $\widetilde{\phi}_{\ell} \equiv i \sigma_{2} \phi_{\ell}^{*}$. In our convention $\phi_{\ell}$ is assigned with the same hypercharge $Y=+1 / 2$ with the SM Higgs doublet $H$ so that $\phi_{\ell}$ can be regarded as the 2nd Higgs doublet in 2HDM. Couplings $\lambda_{-1}$ and $\lambda_{0}$ are not important in our ensuing discussions and are set to be zero. The part involving the two Higgs doublets, as usual, is given by

$$
V_{2 \mathrm{HDM}}=\frac{\lambda_{1}}{2}\left|\phi_{\ell}\right|^{4}+\frac{\lambda_{2}}{2}|H|^{4}+\lambda_{3}\left|\phi_{\ell}\right|^{2}|H|^{2}+\lambda_{4}\left(\phi_{\ell}^{\dagger} H\right)\left(H^{\dagger} \phi_{\ell}\right)+\left(\frac{\lambda_{5}}{2}\left(\phi_{\ell}^{\dagger} H\right)^{2}+\text { h.c. }\right) .
$$


In this potential most parameters are irrelevant to our phenomenological studies, except for $\lambda_{5}$ that is crucial in neutrino mass generation.

A comment deserves special attention. We start from lepton-flavored DM, but as a bonus nonzero neutrino masses are generated as a generic consequence of this type of DM model. It is obvious that all of the crucial ingredients of the Ma's model [14] are incorporated in our framework, and thus radiative corrections lead to neutrino masses:

$$
m_{\nu} \sim \lambda_{5} \frac{y_{L a}^{2}}{16 \pi^{2}}\left(\frac{v}{m_{\phi_{\ell}}}\right)^{2} M
$$

with $v=246 \mathrm{GeV}$. In the parameter space relevant to LFV Higgs decay, $M$ is around the weak scale while $m_{\phi_{\ell}} \sim \mathcal{O}(\mathrm{TeV})$ and moreover $y_{L a} \sim \mathcal{O}(1)$. Then the resulting neutrino mass scale is much above the $\mathrm{eV}$ scale except for extremely suppressed $\lambda_{5} \ll 1$. In this paper we will not pay further attention on this aspect and always assume a sufficiently small $\lambda_{5}$ to suppress radiative neutrino mass.

\subsection{The mass spectrum of the mediators}

In the right vacuum, only $H$ is supposed to develop vacuum expectation value (VEV), breaking the electroweak symmetries but not $Z_{2}$. Then the charged component of $\phi_{\ell}$, which is written in component as $\phi_{\ell}=\left(\phi_{\ell}^{+},\left(\phi_{R}+i \phi_{I}\right) / \sqrt{2}\right)^{T}$, would mix with $\phi_{e}$ through the $\mu$ term, i.e., $\mu v \phi_{e} \phi_{\ell}^{+} / \sqrt{2}+$ c.c.. Then mass eigenstates are related to the flavor eigenstates via

$$
\widetilde{e}_{1}=\cos \theta\left(\phi_{\ell}^{+}\right)^{*}-\sin \theta \phi_{e}, \quad \widetilde{e}_{2}=\sin \theta\left(\phi_{\ell}^{+}\right)^{*}+\cos \theta \phi_{e},
$$

The two charged sleptons respectively have the following (mass) ${ }^{2}$

$$
m_{\tilde{e}_{1,2}}^{2}=\frac{1}{2}\left[\left(m_{\phi_{\ell}}^{2}+m_{\phi_{e}}^{2}\right) \mp \sqrt{\left(m_{\phi_{\ell}}^{2}-m_{\phi_{e}}^{2}\right)^{2}+2 \mu^{2} v^{2}}\right],
$$

respectively. The $\lambda_{3}$-term contributions to masses have been absorbed into the bare mass term of $\phi_{\ell}, m_{\phi_{\ell}}^{2}$ which is common to all components. And similar operation is done for $\phi_{e}$. The mixing angle, within $(-\pi / 2, \pi / 2)$, is given by

$$
\tan \theta=\frac{1}{\sqrt{2} \mu v}\left[\left(m_{\phi_{\ell}}^{2}-m_{\phi_{e}}^{2}\right)+\sqrt{\left(m_{\phi_{\ell}}^{2}-m_{\phi_{e}}^{2}\right)^{2}+2 \mu^{2} v^{2}}\right] .
$$

For completeness, we also give masses for the two neutral components. Their mass degeneracy is lifted by terms in the $V_{2 \mathrm{HDM}}$,

$$
m_{\phi_{R}}^{2} \approx m_{\phi_{\ell}}^{2}+\left(\lambda_{4}+\lambda_{5}\right) v^{2} / 2, \quad m_{\phi_{I}}^{2} \approx m_{\phi_{\ell}}^{2}+\left(\lambda_{4}-\lambda_{5}\right) v^{2} / 2 .
$$

For future convenience, in figure 1 we show the mass ratio $m_{\widetilde{e}_{2}} / m_{\widetilde{e}_{1}}$ and $\tan \theta$ for the cases with a very large and normal $\mu$, respectively.

It is useful to expand the Lagrangian eq. (2.1) in components. For a more general setup, we introduce a Lagrangian that contains a couple of scalar fields $\phi_{\ell}^{+}$with unit charge and 

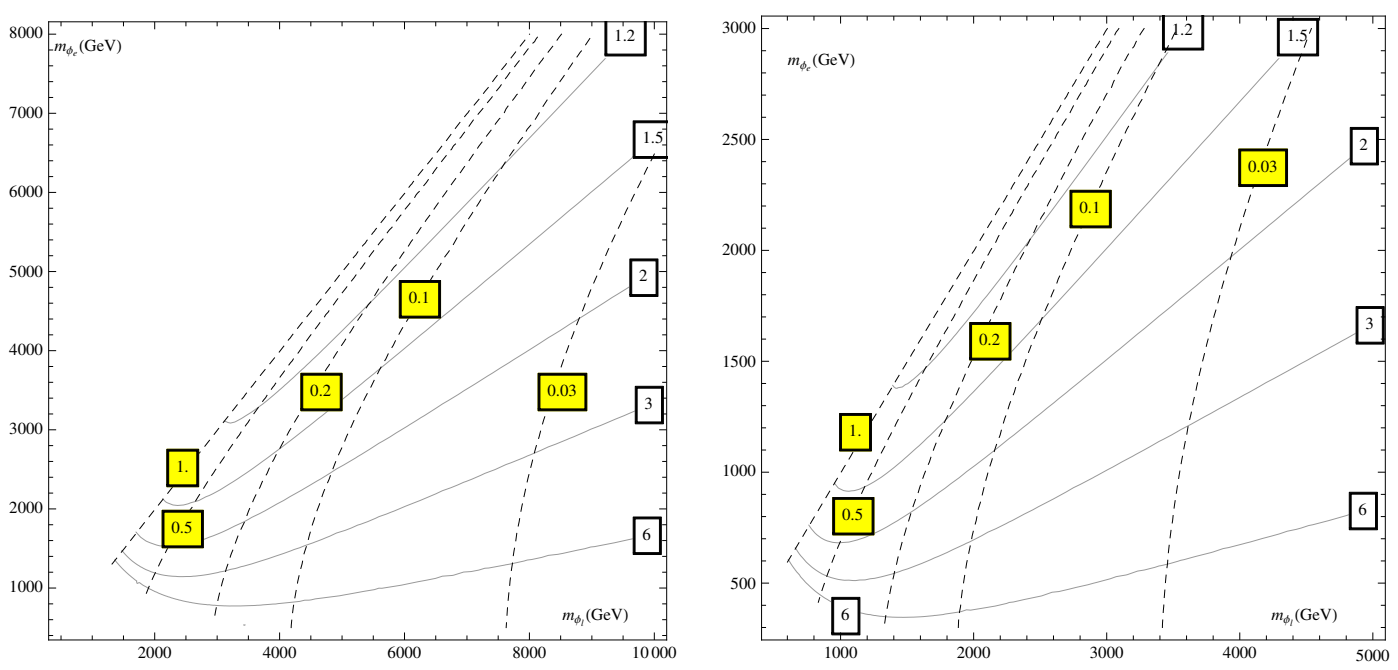

Figure 1. Contour plots of $m_{\widetilde{e}_{2}} / m_{\widetilde{e}_{1}}$ (solid lines) and $\tan \theta$ (solid lines) on the $m_{\phi_{\ell}}-m_{\phi_{e}}$ plane: left $\mu=10 \mathrm{TeV}$; right $\mu=2 \mathrm{TeV}$.

as well several Majorana fermion $N_{\alpha}$ instead of only one (for example in the Ma's model there are three RHNs). Their interactions in the mass basis are given by

$$
\begin{aligned}
-\mathcal{L}= & m_{\widetilde{e}_{i}}^{2}\left|\widetilde{e}_{i}\right|^{2}+\frac{M_{\alpha}}{2} \bar{N}_{\alpha} N_{\alpha}+\frac{1}{2} m_{h}^{2} h^{2} \\
& +A_{i j} h \widetilde{e}_{i}^{*} \widetilde{e}_{j}+\left[\widetilde{e}_{i} \bar{e}_{a}\left(\lambda_{i a \alpha}^{L} P_{L}+\lambda_{i a \alpha}^{R} P_{R}\right) N_{\alpha}+\text { h.c. }\right],
\end{aligned}
$$

with $a=1,2,3$ the generation index. It is assumed that $N_{\alpha}$ 's are Majorana fermions, but practically this assumption is not necessary for generating LFV Higgs decay (but necessary for generating neutrino masses). Expressed in terms of the original parameters, the couplings can be written as

$$
\begin{aligned}
& A_{11}=-A_{22}=-\frac{\mu}{\sqrt{2}} \sin 2 \theta, \\
& A_{12}=A_{21}=\frac{\mu}{\sqrt{2}} \cos 2 \theta,
\end{aligned}
$$

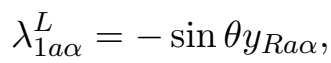

$$
\begin{aligned}
& \lambda_{2 a \alpha}^{L}=\cos \theta y_{R a \alpha} ;
\end{aligned}
$$

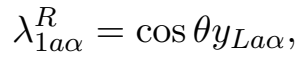

$$
\begin{aligned}
& \lambda_{2 a \alpha}^{R}=\sin \theta y_{L a \alpha},
\end{aligned}
$$

The two neutral sleptons do not play important roles in the following discussions because they do not couple to the Higgs boson with a large massive coupling.

\section{$3 \quad h \rightarrow \tau \mu$ confronting $\tau \rightarrow \mu \gamma$ and $h \rightarrow \gamma \gamma$}

In this section we will investigate how to get large LFV Higgs decay without conflict with the strong constraints such as CLFV or $h \rightarrow \gamma \gamma$. We will concentrate on $h \rightarrow \tau \mu$ as an example, but the discussions can be applied to other similar processes.

\subsection{Radiative LFV Higgs decay: $h \rightarrow \bar{\ell}_{a} \ell_{b}$}

The charged sleptons $\widetilde{e}_{i}$ mediate radiative Higgs LFV decay $h \rightarrow \bar{\ell}_{a} \ell_{b}$, with the Feymann diagram shown in the first panel of figure 2. The corresponding amplitude is generically 

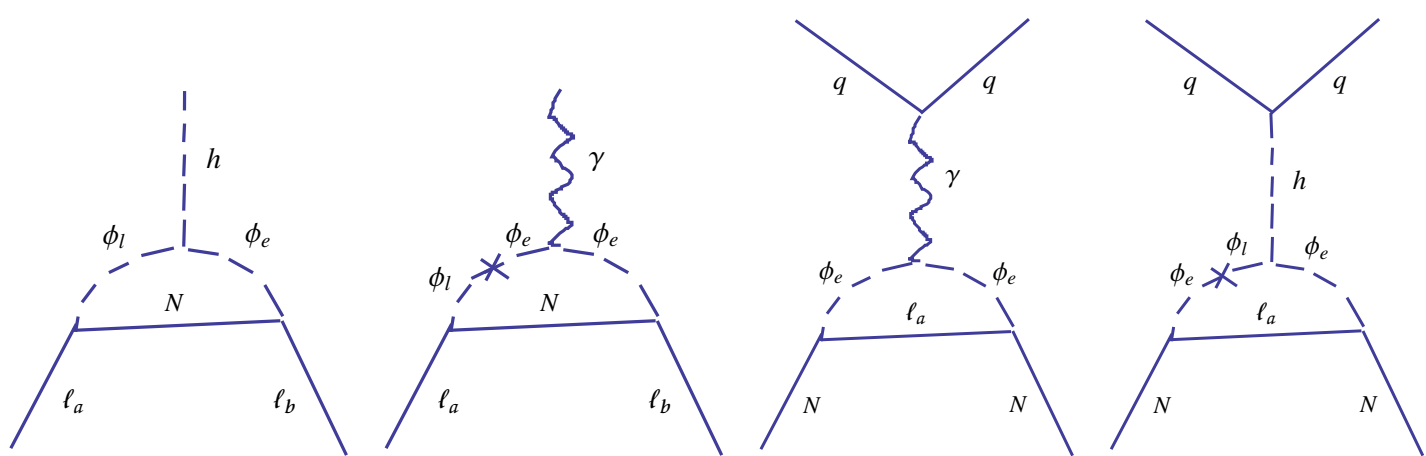

Figure 2. Schematic Feymann diagrams for Higgs (first panel) and charged lepton (second panel) LFV decays; photon (third panel) and Higgs (fourth panel) mediated DM-quark scattering. Loopy particles are in the interacting basis to manifest the dependence on mixing.

written as

$$
i \mathcal{M}=+i \bar{u}_{b}\left(-p_{2}+p_{1}\right)\left(F_{L} P_{L}+F_{R} P_{R}\right) v_{a}\left(p_{2}\right),
$$

with the form factor

$$
\begin{aligned}
F_{L} & =\frac{1}{16 \pi^{2}} M_{\alpha} C_{0}\left(-p_{2}, p_{1}-p_{2}, M_{\alpha}, m_{\widetilde{e}_{i}}, m_{\widetilde{e}_{j}}\right) A_{j i}\left(\lambda_{i a \alpha}^{R}\right)^{*} \lambda_{j b \alpha}^{L} \\
& \simeq \frac{1}{16 \pi^{2}} \frac{\mu}{\sqrt{2} M_{\alpha}} y_{R b \alpha} y_{L a \alpha}^{*}\left[\frac{1}{2} \sin ^{2} 2 \theta\left(G\left(x_{1}\right)+G\left(x_{2}\right)\right)+\cos ^{2} 2 \theta G\left(x_{1}, x_{2}\right)\right],
\end{aligned}
$$

where $x_{i} \equiv m_{\widetilde{e}_{i}}^{2} / M_{\alpha}^{2}$; hereafter, we will consider just one flavor of Majorana, the DM candidate, and thus the index " $\alpha$ " will be implied. To get the above expression, we have neglected the terms proportional to lepton masses, and further assumed $m_{h}^{2} \ll m_{\widetilde{e}_{i}}^{2}, M^{2}$ in the last line. $F_{R}$ can be obtained simply by exchanging $L \leftrightarrow R$. We emphasize that to get eq. (3.2) which is not suppressed by small lepton masses we need both left- and righthanded scalar mediators, which can be seen obviously from the fact it is proportional to $\mu$-parameter (See eq. (2.1) and also the first panel of figure 2). The term with $\sin ^{2} 2 \theta$ comes from the contributions of $\widetilde{e}_{1}-\widetilde{e}_{1}$ and $\widetilde{e}_{2}-\widetilde{e}_{2}$, while the term with $\cos ^{2} 2 \theta$ comes from those of $\widetilde{e}_{1}-\widetilde{e}_{2}$ contributions in the loop. If we had a mediator with only one chirality, the chirality flip required in eq. (3.1) would occur only in external lepton lines. As a consequence the amplitude would be suppressed by small lepton masses and we could not get sizable $h \rightarrow \mu \tau$ rate. In this paper, we follow the notations of three-point scalar function $C_{0}$ as in ref. [42]. The loop functions $G\left(x_{1}, x_{2}\right)=G\left(x_{2}, x_{1}\right)$ and $G\left(x_{1}\right) \equiv G\left(x_{1}, x_{1}\right)$ are defined in eq. (A.3) and eq. (A.4), respectively.

As expected, in the decoupling limit with $\theta \rightarrow 0$ (or $\pi / 2$ ), the first term of $F_{L}$ is suppressed. In contrast, in the maximal mixing limit $\theta \rightarrow \pi / 4$, the second term is suppressed. Later, the former feature will be utilized to suppress LFV decay of charged leptons.

The decay width of $h \rightarrow \bar{\ell}_{a} \ell_{b}$ is calculated as

$$
\Gamma\left(h \rightarrow \bar{\ell}_{a} \ell_{b}\right)=\frac{m_{h}}{16 \pi}\left(\left|F_{L}\right|^{2}+\left|F_{R}\right|^{2}\right) .
$$




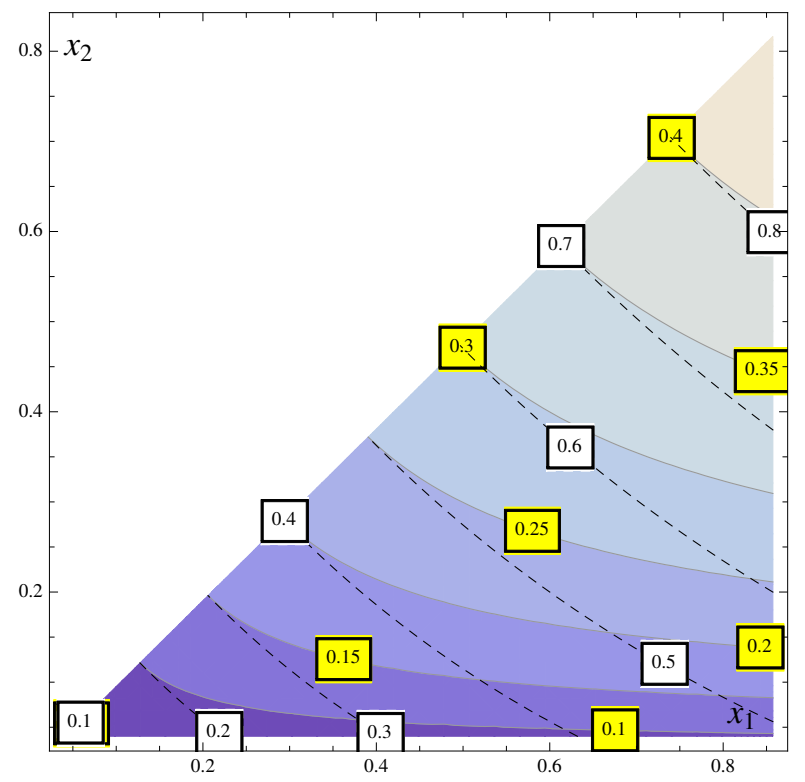

Figure 3. Contour plots of loop functions $G\left(x_{1}^{-1}, x_{2}^{-1}\right)$ (solid lines) and $G\left(x_{1}^{-1}\right)+G\left(x_{2}^{-1}\right)$ (dashed lines). In the plot we use the variables $1 / x_{i}$ instead of $x_{i}$; the same convention applies to other figures.

For concreteness, we take $\ell_{a}=\tau$ and $\ell_{b}=\mu$ hereafter. In addition, for simplicity we consider only one chiral structure, i.e., setting $y_{L \tau}=y_{R \mu}=0$. It is easy to recover the corresponding contributions by the replacement $L \rightarrow R$ and $R \rightarrow L$ for all the later expressions. The implication of relaxation of this assumption will be commented when necessary. For reference, the branching ratio of $h \rightarrow \bar{\tau} \mu$ is estimated in those two limits, the decoupling limit $(\theta \rightarrow 0)$ :

$$
\operatorname{Br}(h \rightarrow \bar{\tau} \mu)=1.2 \times 10^{-2}\left(\frac{\mu}{5 \mathrm{TeV}}\right)^{2}\left(\frac{1 \mathrm{TeV}}{M}\right)^{2}\left(\frac{G\left(x_{1}, x_{2}\right)}{0.2}\right)^{2}\left(\frac{\left|y_{R \tau} y_{L \mu}^{*}\right|}{1}\right)^{2} ;
$$

and the maximal mixing limit $(\theta \rightarrow \pi / 4)$ :

$$
\operatorname{Br}(h \rightarrow \bar{\tau} \mu)=1.2 \times 10^{-2}\left(\frac{\mu}{10 \mathrm{TeV}}\right)^{2}\left(\frac{1 \mathrm{TeV}}{M}\right)^{2}\left(\frac{G\left(x_{1}\right)+G\left(x_{2}\right)}{0.4}\right)^{2}\left(\frac{\left|y_{R \tau} y_{L \mu}^{*}\right|}{0.5}\right)^{2}
$$

The total decay width of Higgs boson has been taken to be $4 \mathrm{MeV}$. We show contour plots of $G\left(x_{1}, x_{2}\right)$ and $G\left(x_{1}\right)+G\left(x_{2}\right)$ in figure 3 .

\subsection{Induced CLFV $\tau \rightarrow \mu \gamma$}

The LFV decays of charged leptons are good probes to LFV. For example, the present experimental upper bound on $\operatorname{Br}(\tau \rightarrow \mu \gamma)$ is $4.4 \times 10^{-8}$ [43] at $90 \%$ C.L. and will be improved by one order of magnitude in the near future [44]. The upper bound on CLFV decay of muon is even more stringent, $\operatorname{Br}(\mu \rightarrow e \gamma)<5.7 \times 10^{-13}$ at $90 \%$ C.L., from the current MEG result [45]. On the other hand, LFV Higgs decay is likely to induce CLFV decay (but not vice versa). Illustratively, the Feynman diagrams of the latter can 
be obtained simply by replacing the Higgs field with a photon leg in the charged loop of the diagram for the former. As a schematic example, see the first and second panels of figure 2. Since both processes share almost the same loops, a hierarchical ratio $\operatorname{Br}(h \rightarrow$ $\tau \mu) / \operatorname{Br}(\tau \rightarrow \mu \gamma)$ as large as $10^{5}$ then raises doubt.

LFV decay of $\tau$ into $\mu+\gamma$ can be generically described by the following effective Hamiltonian:

$$
\mathcal{H}_{\mathrm{eff}}=C_{L} \overline{\mu_{L}} \sigma^{\mu \nu} \tau_{R} F_{\mu \nu}+C_{R} \overline{\mu_{R}} \sigma^{\mu \nu} \tau_{L} F_{\mu \nu}
$$

Different to significant chirality flip by virtue of the Higgs field in the loop of LFV Higgs decay process, here vector current conserves chirality. There are three other chirality violation sources to generate the Wilsonian coefficients $C_{L, R}$,

$$
\begin{aligned}
C_{L}=\frac{e}{32 \pi^{2}}[\{ & \left.\frac{m_{\mu}}{M^{2}} y_{R \mu} y_{R \tau}^{*}\left(s_{\theta}^{2} F_{1}\left(x_{1}\right)+c_{\theta}^{2} F_{1}\left(x_{2}\right)\right)+\frac{m_{\tau}}{M^{2}} y_{L \mu} y_{L \tau}^{*}\left(c_{\theta}^{2} F_{1}\left(x_{1}\right)+s_{\theta}^{2} F_{1}\left(x_{2}\right)\right)\right\} \\
& \left.-\frac{1}{M} y_{L \mu} y_{R \tau}^{*} s_{\theta} c_{\theta}\left(F_{2}\left(x_{1}\right)-F_{2}\left(x_{2}\right)\right)\right] .
\end{aligned}
$$

The expression of $C_{R}$ can be obtained via $L \leftrightarrow R$. The loop functions $F_{1}(x)$ and $F_{2}(x)$ are defined as

$$
\begin{aligned}
& F_{1}(x)=\frac{2+3 x-6 x^{2}+x^{3}+6 x \log x}{12(1-x)^{4}} \\
& F_{2}(x)=\frac{-1+x^{2}-2 x \log x}{2 x(1-x)^{2}} .
\end{aligned}
$$

According to the Hamiltonian, the decay width of $\tau \rightarrow \mu \gamma$ after summing over polarizations is calculated to be

$$
\Gamma(\tau \rightarrow \mu \gamma)=\frac{\left(m_{\tau}^{2}-m_{\mu}^{2}\right)^{3}}{4 \pi m_{\tau}^{3}}\left[\left|C_{L}\right|^{2}+\left|C_{R}\right|^{2}\right]
$$

In $C_{L}$, the first and the second terms do not require the simultaneous presence of $y_{L}$ and $y_{R}$ because chirality flip comes from the external lines, i.e., the Dirac mass term of lepton. But they require LFV through the same chirality of slepton. These contributions are generically subdominant, compared to the third term, given a large $M$ and as well democratic type Yukawa coupling, i.e., $y_{L} \sim y_{R}$. Besides, a sizable mixing angle between $\phi_{\ell}^{+}$and $\phi_{e}$ is needed. This means that, not only flavor violation but also chirality violation are provided by the sleptons, as is well understood from the second panel of figure 2 .

We argue that the $h \rightarrow \tau \mu$ rate can be enhanced while suppressing $\tau \rightarrow \mu \gamma$. (See section 3.4 for more details.) One obvious mechanism is to use heavy $\phi_{e}$, which naturally leads to small mixing angle $\theta$. In this case the $\tau \rightarrow \mu \gamma$ diagram has one more $\phi_{e}$ propagator compared with the $h \rightarrow \tau \mu$ diagram as shown in the first two diagrams in figure 2 , suppressing the former compared to the latter. 


\subsection{Hints in $h \rightarrow \gamma \gamma$}

Since LFV Higgs decay heavily depends on the charged scalar mixing term, $h \rightarrow \gamma \gamma$ inevitably receives a sizable contribution. Under the assumption that other Higgs decay modes are not affected, which is a very natural assumption, we get the modification to $h \rightarrow \gamma \gamma$ from the $\widetilde{e}_{1}$-loop $[46],{ }^{2}$

$$
c_{\gamma}=c_{\mathrm{SM}, \gamma}+\delta c_{\gamma} \approx-0.81-\frac{1}{24} \frac{v \mu \sin 2 \theta}{2 \sqrt{2} m_{\widetilde{e}_{1}}^{2}}
$$

Here $c_{\gamma}$ denotes the reduced coupling of the dimension-five operator for coupling between Higgs and photons, $c_{\gamma} \frac{\alpha}{\pi v} h F_{\mu \nu} F^{\mu \nu}$. The sign of $\mu$ is indeterminate, so one can make $r_{\gamma}$ close to the SM value either by requiring a small $\delta c_{\gamma} \ll 1$ or $\delta c_{\gamma} \sim+1.62$, which flips the sign of $c_{\gamma}$ relative to the SM one. To be more specific, we refer to a recent study [48], from which we know that at $68.3 \%$ C.L. there are two allowed regions:

$$
-0.05 \lesssim \delta c_{\gamma} / c_{\mathrm{SM}, \gamma} \lesssim 0.20, \quad-2.20 \lesssim \delta c_{\gamma} / c_{\mathrm{SM}, \gamma} \lesssim-1.95
$$

Feeding these results back to the slepton sector we get the following constraints:

- In the first region, one gets the bounds:

$$
-1.0 \times\left(\frac{m_{\widetilde{e}_{1}}}{300 \mathrm{GeV}}\right)^{2} \mathrm{TeV} \lesssim \mu \sin 2 \theta \lesssim 4.0 \times\left(\frac{m_{\widetilde{e}_{1}}}{300 \mathrm{GeV}}\right)^{2} \mathrm{TeV} .
$$

As one can see, as long as $\widetilde{e}_{1}$ mass is at least a few hundred GeVs, the Higgs diphoton rate in the decoupling limit can be easily suppressed below the upper bound. But it is not that easy to reconcile $\operatorname{Br}(h \rightarrow \tau \mu)$ and $\operatorname{Br}(h \rightarrow \gamma \gamma)$ in the maximal mixing limit. The $\widetilde{e}_{1}$ should be sufficiently heavy, or it should have roughly equal mass with $\widetilde{e}_{2}$ in the light of footnote 2 .

- The second region allows for the scenarios with a huge $\mu$ along with a lighter $m_{\widetilde{e}_{1}}$. In this way of reconciling $\operatorname{Br}(h \rightarrow \tau \mu)$ and $\operatorname{Br}(h \rightarrow \gamma \gamma)$, it (asides from determining the sign of $\mu$ ) actually helps to eliminate one of the three parameters in the slepton sector:

$$
\mu \sin 2 \theta \approx 40.2 \times\left(\frac{m_{\widetilde{e}_{1}}}{300 \mathrm{GeV}}\right)^{2}\left(\frac{\delta c_{\gamma}}{-2.0 c_{\mathrm{SM}, \gamma}}\right) \mathrm{TeV}
$$

A TeV scale $m_{\widetilde{e}_{1}}$ will blow up $\mu$, thus disfavored. By the way, a too large $\mu / m_{\widetilde{e}_{1}} \gg 10$ may also change Higgs self-coupling too much.

In summary, Higgs diphoton does not give a severe constraint. But it is interesting to see that possibly the rate can be related to the large LFV Higgs decay.

\footnotetext{
${ }^{2}$ In the following analysis we decouple the $\widetilde{e}_{2}$-loop by assuming much heavier $\widetilde{e}_{2}$, otherwise the contributions from the $\widetilde{e}_{1}$ - and $\widetilde{e}_{2}$-loop show substantial cancellation: $\delta c_{\gamma} \approx-\frac{v \mu \sin 2 \theta}{48 \sqrt{2}}\left(\frac{1}{m_{\widetilde{e}_{1}}^{2}}-\frac{1}{m_{\widetilde{e}_{2}}^{2}}\right)$. Then the bound becomes weaker.
} 


\subsection{Natural ways to get large $h \rightarrow \tau \mu$}

We have collected all the necessary formulas to calculate $\operatorname{Br}(h \rightarrow \tau \mu)$ under the constraints such as $\operatorname{Br}(\tau \rightarrow \mu \gamma)$. In this subsection we show how $\operatorname{Br}(h \rightarrow \tau \mu) \sim 10^{-2}$ can be realized. For that, it is convenient to study the ratio $R_{\tau} \equiv \operatorname{Br}(h \rightarrow \tau \mu) / \operatorname{Br}(\tau \rightarrow \mu \gamma)$. To explain the central value of the $h \rightarrow \tau \mu$ signal, $B(h \rightarrow \tau \mu) \approx 0.85 \%$, with the contraint $B(\tau \rightarrow$ $\mu \gamma)<4.4 \times 10^{-8}$, we need $R_{\tau} \gtrsim 2 \times 10^{5}$. In the decoupling limit of the scalar system, $R_{\tau}$ can be illustratively parameterized as

$$
R_{\tau} \approx 2.8 \times 10^{5}\left(\frac{\mu}{10 \mathrm{TeV}}\right)^{2}\left(\frac{0.1}{\sin \theta}\right)^{2}\left(\frac{G\left(x_{1}, x_{2}\right) /\left(F_{2}\left(x_{2}\right)-F_{2}\left(x_{1}\right)\right)}{20}\right)^{2} .
$$

We have made the approximation that eq. (3.2) and eq. (3.7) are dominated by the second and third terms, respectively. In this approximation, $R_{\tau}$ is independent of (or insensitive to) the following parameters: (I) DM mass $M$; (II) the Yukawa couplings; (III) to some degree, also $\mu$. To see the last point, from eq. (2.6) one may have $1 / \sin 2 \theta \approx m_{\widetilde{e}_{2}}^{2} / \sqrt{2} \mu \nu$ and consequently $\mu^{2}$ is cancelled. This conclusion holds for a well asymmetric scalar system like $m_{\phi_{\ell}}^{2} \gg m_{\phi_{e}}^{2}, 2 \mu^{2} v^{2}$, which guarantees decoupling scalars as desired. If instead the scalar sector is in the maximal mixing limit and thus eq. (3.2) is dominated by the first term, we have the estimation

$$
R_{\tau} \approx 2.8 \times 10^{5}\left(\frac{\mu}{10 \mathrm{TeV}}\right)^{2}\left(\frac{\left(G\left(x_{1}\right)+G\left(x_{2}\right)\right) /\left(F_{2}\left(x_{2}\right)-F_{2}\left(x_{1}\right)\right)}{200}\right)^{2} .
$$

In the absence of enhancement from (the inverse of) small mixing, one needs a huge $\mu$ at least $10 \mathrm{TeV}$ and at the same time a very large ratio $\left(G\left(x_{1}\right)+G\left(x_{2}\right)\right) /\left(F_{2}\left(x_{2}\right)-F_{2}\left(x_{1}\right)\right) \sim$ $\mathcal{O}(100)$. While in the previous case it is moderate. That large ratio may incur a significant fine-tuning. In order to lift the ratio, one needs cancelation ${ }^{3}$ between $F_{2}\left(x_{2}\right)$ and $F_{2}\left(x_{1}\right)$. Obviously, if $x_{1} \approx x_{2}$, cancelation happens. ${ }^{4}$

Regarding the difference $F_{2}\left(x_{2}\right)-F_{2}\left(x_{1}\right)$ as a function of three fundamental variables $\mu_{i}=\left(m_{\widetilde{e}_{1}}, m_{\widetilde{e}_{2}}, M\right)$, we can measure fine-tuning using the quantity

$$
\Delta=\left.\max \left\{\left|\Delta_{i}\right|\right\}\right|_{i=1,2,3} \text { with } \Delta_{i} \equiv \frac{\partial \log \left(F_{2}\left(x_{2}\right)-F_{2}\left(x_{1}\right)\right)}{\partial \log \mu_{i}} .
$$

Explicitly, $\Delta_{i}=2\left(c_{i 2} x_{2} F_{2}^{\prime}\left(x_{2}\right)-c_{i 1} x_{1} F_{2}^{\prime}\left(x_{1}\right)\right) /\left(F_{2}\left(x_{2}\right)-F_{2}\left(x_{1}\right)\right)$ with $c_{11,12}=(1,0)$, $c_{21,22}=(0,1)$ and $c_{31,32}=(-1,-1)$.

Let us denote the ratio of loop functions in eq. (3.14) and eq. (3.15) as $r\left(x_{1}, x_{2}\right)$. In figure 4 , we plot the distributions of $r\left(x_{1}^{-1}, x_{2}^{-1}\right)$ and fine-tuning $\Delta$ on the $x_{1}-x_{2}$ plane. The left and the right panel are for the decoupling and the maximal mixing scenarios, respectively. The shaded regions have degree of fine-tuning less than $5 \%$, which is referred as the lower bound for naturalness in this article. It is seen that the decoupling scenario

\footnotetext{
${ }^{3}$ Ref. [19] also considered cancelations in $\tau \rightarrow \mu \gamma$ via introducing some extra contributions to cancel the contribution induced by $h \rightarrow \tau \mu$. In our model this is kind of cancelation happens within well expectation.

${ }^{4}$ Cancelation also happens for $x_{1} \neq x_{2}$. In particular, for a (at least) mild mass hierarchy between $m_{\widetilde{e}_{1}}^{2}$ and $m_{\tilde{e}_{2}}^{2}$, cancelation approximately determines $M: M \simeq \frac{m_{\tilde{e}_{1}} m_{\tilde{e}_{2}}}{\sqrt{3 m_{\tilde{e}_{1}}^{2}+m_{\tilde{e}_{2}}^{2}}}$.
} 

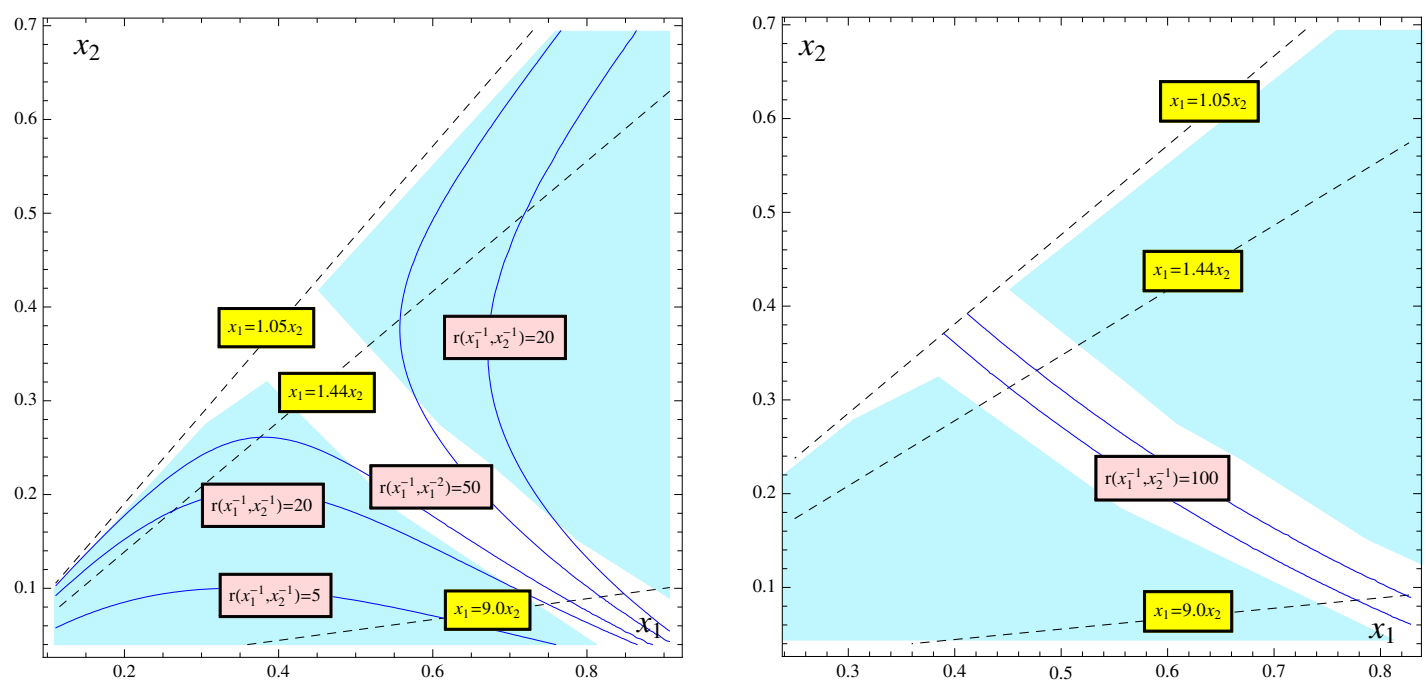

Figure 4. Contour plots of loop functions ratio $r\left(x_{1}^{-1}, x_{2}^{-1}\right)$ (blue lines), which is $G\left(x_{1}^{-1}, x_{2}^{-1}\right) /\left(F_{2}\left(x_{2}^{-1}\right)-F_{2}\left(x_{1}^{-1}\right)\right.$ in the decoupling limit (left) and $\left(G\left(x_{1}^{-1}\right)+G\left(x_{2}^{-1}\right)\right) /\left(F_{2}\left(x_{2}^{-1}\right)-\right.$ $F_{2}\left(x_{1}^{-1}\right)$ in the maximal mixing limit (right panel). Regions with fine-tuning better than $5 \%$ are shaded. Besides, we label three selected ratios of the masses of two charged scalars (dashed lines).

can provide $r\left(x_{1}^{-1}, x_{2}^{-1}\right) \sim \mathcal{O}(10)$ barely incurring fine-tuning; in contrast, the maximal mixing scenario, which needs $r\left(x_{1}^{-1}, x_{2}^{-1}\right) \gtrsim 100$, typically incurs fine-tuning worse than $5 \%$. But the cancelation via degenerate $\widetilde{e}_{1}$ and $\widetilde{e}_{2}$ still opens a narrow region around the point $x_{1}^{-1} \simeq x_{2}^{-1} \sim 0.6$ or closely alone the line $x_{1}=x_{2}$, which without a particular UV reason is not of much interest. In what follows we will focus on these two kinds of natural regions.

Let us consider the decoupling scenario. We make several observations that are helpful to trace back to the patterns of scalar mass squared matrix.

1. If both $x_{1,2}^{-1} \ll 1$, we need significant degeneracy between two scalars, see the leftbottom corner of the left panel of figure 4. Since we are chasing the decoupling limit confronting a large slepton mixing term with $\mu \sim \mathcal{O}(10) \mathrm{TeV}$, this means large and degenerate scalar mass terms $m_{\phi_{\ell}}^{2} \sim m_{\phi_{e}}^{2} \gg \mathcal{O}(1) \mathrm{TeV}^{2}$. It results in a heavy spectrum typically having multi-TeV sleptons, see the left panel of figure 1.

2. There is a hierarchy $x_{2}^{-1} \lesssim \mathcal{O}(0.1) x_{1}^{-1}$, keeping $x_{1}^{-1}$ close to 1 . It requires an asymmetric scalar system, e.g., $m_{\phi_{\ell}}^{2} \gg m_{\phi_{e}}^{2} \sim \mathcal{O}(1) \mathrm{TeV}^{2}$, the most favored pattern to decouple $\phi_{\ell}$ and $\phi_{e}$ with a large mixing term.

3. $x_{2}^{-1} \lesssim x_{1}^{-1}$, both not far from 1 . This is in the bulk space without special requirements. Even for a smaller $\mu$ near the $\mathrm{TeV}$ scale, one is still able to produce such a case readily, yielding a lighter spectrum inducing DM.

In summary, there is a wide parameter space for the decoupling scenario. In practice, in some situations the mixing angle is supposed to be moderately small rather than very small. 


\section{Leptophilic DM: relic density \& direct detection}

The DM candidate $N,^{5}$ is a singlet Majorana fermion with $t$-channel mediators, and its phenomenologies in some simplified cases have been investigated compressively in ref. [49]. But our case turns out to be significantly different, due to the appearance of both $\phi_{\ell}$ and $\phi_{e}$ mediators. In this section we will focus on two main differences, annihilation and direct detection of DM.

\subsection{Annihilation: $s$-wave versus $p$-wave}

The first difference comes from DM annihilating. The Majorana DM $N$ annihilates into leptons through the interactions given in eq. (2.8). They proceed with $\widetilde{e}_{i}$ exchanging in the $t$ - and $u$-channel. We can calculate the cross section expanded in terms of DM relative velocity $v_{r} \equiv 2 \sqrt{1-4 M^{2} / s}$ in the center-of-mass (CM) frame: $\sigma v_{r} \approx a+b v_{r}^{2}$ with the $s$ and $p$-wave coefficients respectively given by

$$
\begin{aligned}
a & =\frac{1}{16 \pi M^{2}} \frac{1}{\left(1+x_{i}\right)^{2}}\left(\left|\lambda_{i a}^{L} \lambda_{i b}^{R}\right|^{2}+\left|\lambda_{i a}^{R} \lambda_{i b}^{L}\right|^{2}\right), \\
b & =\frac{1}{96 \pi M^{2}} \frac{1}{\left(1+x_{i}\right)^{4}}\left[2\left|\lambda_{i a}^{L}\right|^{2}\left|\lambda_{i b}^{L}\right|^{2}\left(1+x_{i}\right)-\left|\lambda_{i a}^{L}\right|^{2}\left|\lambda_{i b}^{R}\right|^{2}\left(1+4 x_{i}-3 x_{i}^{2}\right)+(L \leftrightarrow R)\right] .
\end{aligned}
$$

The inclusive annihilation rate should sum over the family index $a$ and $b$. As a check, when the model goes to the chiral limit considered previously [49], e.g., $\lambda_{i}^{R}$ (or $y_{L}$ ) $\rightarrow 0$, we recover the well known result: $a=0$ (up to contributions suppressed by lepton masses). Then, DM must annihilate away mainly via $p$-wave, whose coefficient takes a form of

$$
b \rightarrow \frac{1}{48 \pi M^{2}} \frac{1+x_{i}}{\left(1+x_{i}\right)^{4}}\left|\lambda_{i a}^{L} \lambda_{i b}^{L}\right|^{2} .
$$

It is not suppressed by small mixing. For instance $\theta \rightarrow 0$, it still receives a contribution from $\left|\lambda_{2 a}^{L} \lambda_{2 b}^{L}\right|^{2} \rightarrow\left|y_{R a} y_{R b}\right|^{2}$. With them, the relic density can be calculated via the well-known formula [50]

$$
\Omega h^{2} \approx \frac{0.88 \times 10^{-10} x_{f} \mathrm{GeV}^{-2}}{g_{*}^{1 / 2}\left(a+3 b / x_{f}\right)} .
$$

At the freeze-out epoch $x_{f}=M / T_{f} \sim 20$, the effective degree of freedom $g_{*} \sim 100$. If we demand the Yukawa coupling constants $\lesssim \mathcal{O}(1)$, in order to maintain perturbativity of the model up to a very high scale, then both DM and mediators should around the weak scale. This is a strong requirement and yields deep implication to direct detection.

But here the $s$-wave may be sufficient to reduce the DM number density, even facing the stringent CLFV constraint and at the same time satisfying the tentative LFV Higgs

\footnotetext{
${ }^{5}$ In our model, in principle DM can be either the neutral component of Higgs doublet $\phi_{\ell}$ or the Majorana fermion $N$. But only the fermonic DM could be a natural leptophilic DM.
} 
decay. It is seen that the $s$-wave coefficient is directly correlated with CLFV decay width $\Gamma\left(\ell_{a} \rightarrow \ell_{b} \gamma\right)$, see eq. (3.7). To be more specific, we write $a$ in terms of others

$$
a \approx \frac{1}{64 \pi M^{2}} \sin ^{2} 2 \theta\left(\left|y_{L a} y_{R b}\right|^{2}+\left|y_{R a} y_{L b}\right|^{2}\right) \sum_{i} \frac{1}{\left(1+x_{i}\right)^{2}} .
$$

It may reach the typical cross section of thermal $\mathrm{DM}, 1 \mathrm{pb}$. To see this, we parameterize the order of magnitude of $a$ as the following:

$$
a \simeq 0.8 \times\left(\frac{400 \mathrm{GeV}}{M}\right)^{2}\left(\frac{\sin ^{2} \theta}{0.1}\right) \frac{\left(\left|y_{L a} y_{R b}\right|^{2}+\left|y_{R a} y_{L b}\right|^{2}\right)}{1.0} \mathrm{pb} .
$$

We have taken $1 /\left(1+x_{1}\right)^{2} \approx 0.15$. Therefore, again a weak scale DM along with (at least one) weak scale mediator can lead to correct relic density via $s$-wave annihilation as long as the mixing angle is not highly suppressed.

Although the $s$-wave annihilation readily works for flavors like $a=b$ which does not violate lepton flavor, it fails for the case under consideration $a=3, b=2$ or inverse. Let us show it in the decoupling scenario. With the aid of eq. (3.4) and eq. (3.14) we can express $a$ as (aside from the propagator factor)

$$
a \simeq 2.1 \times\left(\frac{\operatorname{Br}(h \rightarrow \tau \mu)}{10^{-2}}\right)\left(\frac{3 \times 10^{5}}{R_{\tau}}\right)\left(\frac{10^{-4}}{F_{2}\left(x_{2}\right)-F_{2}\left(x_{1}\right)}\right)^{2} \mathrm{pb} .
$$

But that small value of $F_{2}\left(x_{2}\right)-F_{2}\left(x_{1}\right)$ either incurs large fine-tuning or should follow closely the line $x_{1}^{-1} \simeq x_{2}^{-1} \ll 0.1$. The latter leads to additional suppression $\sim 1 / x_{1}^{2}$ (it has been fixed to be 0.15 in the above estimation). Similarly, the maximal mixing scenario fails either.

We make a comment on the coannihilation effect [50]. Despite of not a focus here, it has two interesting points. First, mass degeneracy between $\widetilde{e}_{1}$ and $M$ is well consistent with the suppression of $\operatorname{Br}(\tau \rightarrow \mu \gamma)$, which is made small by the cancellation mechanism with $x_{1} \neq x_{2}$. For a strong mass hierarchy case $m_{\widetilde{e}_{2}}^{2} \gg m_{\widetilde{e}_{1}}^{2}$, from footnote 4 we have $M \approx m_{\widetilde{e}_{1}}$. Second, by virtue of a large $\mu$-term, the effective cross section of coannihilation is enhanced by the process $\widetilde{e}_{1}^{+} \widetilde{e}_{1}^{-} \rightarrow h h$ with $\widetilde{e}_{1}$ in the $t$-channel:

$$
\begin{aligned}
\sigma_{h h} v & \approx \frac{1}{64 \pi} \frac{1}{m_{\widetilde{e}_{1}}^{2}}\left(\frac{\mu^{2} / 2}{m_{\widetilde{e}_{1}}^{2}}\right)^{2} \\
& =1.2 \times 10^{-5}\left(\frac{1 \mathrm{TeV}}{m_{\widetilde{e}_{1}}}\right)^{2}\left(\frac{\mu / m_{\widetilde{e}_{1}}}{10}\right)^{4} \mathrm{GeV}^{-2}
\end{aligned}
$$

We have worked in the maximal mixing $\sin 2 \theta \approx 1$. So, once $\mu \sim \mathcal{O}(10 \mathrm{TeV})$, the enhancing factor still scales as $\left(\mu / m_{\widetilde{e}_{1}}\right)^{4} \sim \mathcal{O}\left(10^{4}\right)$ even for a TeV scale $m_{\widetilde{e}_{1}}$.

\subsection{On (in)direct searches for the leptophilic DM}

We have shown that DM can gain correct relic density readily. And DM mass should be around the weak scale so as to avoid large Yukawa couplings. In this subsection we move 
to the second difference, direct detection. As a leptophilic DM, DM-nucleon scattering is absent at tree level, but could be generated by radiative corrections. There are two types of corrections leading to DM-nucleon scattering, one mediated by photon and the other Higgs boson, respectively. In particular, the second type, which is absent in the previous setup, benefits from $\mu$-enhancement and can potentially overcome the loop suppression.

The second type is the usual dimension-four operator which comes from the vertex correction on $h \bar{N} N$, absent at tree level but generated after EWSB. In the DM direct detection, typically the transferring momentum $Q^{2}$ is very small compared with the other mass scales in the charged particles in the loops, so that

$$
\mathcal{O}_{h}=\lambda_{h N}(0) h \bar{N} N
$$

where $h$ is treated off-shell with invariant mass $Q^{2} \ll m_{h}^{2}$. The effective coupling at zero momentum transfer is expressed as

$$
\begin{aligned}
\lambda_{h N}(0) \approx \sin \theta \frac{\left|y_{L a}\right|^{2}+\left|y_{R a}\right|^{2}}{32 \pi^{2}} \frac{\mu}{\sqrt{2} M}\left[B_{0}\left(p_{1}-p_{2}\right)_{a 1}-B_{0}\left(p_{1}-p_{2}\right)_{a 2}\right. \\
-2 B_{0}\left(p_{1}\right)_{11}+2 B_{0}\left(p_{1}\right)_{12}-C_{0}\left(-p_{2}, p_{1}-p_{2}\right)_{a 12}\left(m_{\widetilde{e}_{1}}^{2}+m_{\widetilde{e}_{2}}^{2}-2 M^{2}\right) \\
\left.+2 C_{0}\left(-p_{2}, p_{1}-p_{2}\right)_{a 11}\left(m_{\widetilde{e}_{1}}^{2}-M^{2}\right)\right] .
\end{aligned}
$$

Using the kinematics and the approximations of two- and three-point scalar functions in appendix A, we can further simplify it into

$$
\begin{gathered}
\lambda_{h N}(0) \approx \sin \theta \frac{\left|y_{L a}\right|^{2}+\left|y_{R a}\right|^{2}}{32 \pi^{2}} \frac{\mu}{\sqrt{2} M}\left[2+\left(x_{1}-1\right) \log \left(1-x_{1}^{-1}\right)-\left(x_{2}-1\right) \log \left(1-x_{2}^{-1}\right)\right. \\
\left.-\frac{1-x_{1}^{-1}}{1-x_{2}^{-1}} \log \frac{x_{2}}{x_{1}}-\mathcal{G}\left(x_{1}, x_{2}\right)\left(x_{2}+x_{1}-2\right)+2 \mathcal{G}\left(x_{1}, x_{1}\right)\left(x_{1}-1\right)\right],
\end{gathered}
$$

with $\mathcal{G}\left(x_{1}, x_{2}\right)$ seen in eq. (B.5). Note that $x_{1} \simeq x_{2}$ shows cancellation and thus larger $\lambda_{h N}(0)$ dwells on the region with $x_{1}$ at least modularity larger than $x_{2}$.

The Higgs mediated DM-nucleon scattering has a spin-independent cross section $\sigma_{\mathrm{SI}}^{p}=$ $4 m_{p}^{2} f_{p}^{2} / \pi$ with $f_{p}$ given by

$$
f_{p}=\frac{\lambda_{h N}(0)}{m_{h}^{2}} m_{p}\left[\sum_{q=u, d, s} y_{q} \frac{f_{T_{q}}^{(p)}}{m_{q}}+\frac{2}{27} f_{T_{G}}^{(p)} \sum_{q=c, b, t} \frac{y_{q}}{m_{q}}\right] .
$$

In this paper we take $f_{T_{u}}^{(p)}=0.020, f_{T_{d}}^{(p)}=0.026, f_{T_{s}}^{(p)}=0.118$ and $f_{T_{G}}^{(p)}=0.840[51]$ (For more discussions about the calculation and uncertainties of these values, see refs. [52, 53]) and we then get the estimation $\sigma_{\mathrm{SI}}^{p} \approx 4.0 \times 10^{-8}\left(\lambda_{h N}(0) / 0.1\right)^{2} \mathrm{pb}$, a value near the sensitivity of the current LUX. In the bulk parameter space, $\lambda_{h N}(0) \lesssim \mathcal{O}(0.01)$ :

$$
\lambda_{h N}(0) \approx 0.01 \times\left(\frac{\sin \theta}{0.2}\right)\left(\frac{\left|y_{L a}\right|^{2}+\left|y_{R a}\right|^{2}}{1}\right)\left(\frac{\mu}{5 \mathrm{TeV}}\right)\left(\frac{0.3 \mathrm{TeV}}{M}\right) .
$$

The decoupling scenario is hard to be probed, but the maximal mixing scenario, which badly needs a very large $\mu$, has a good prospect. We choose a benchmark case which is directly related with $h \rightarrow \tau \mu$. 


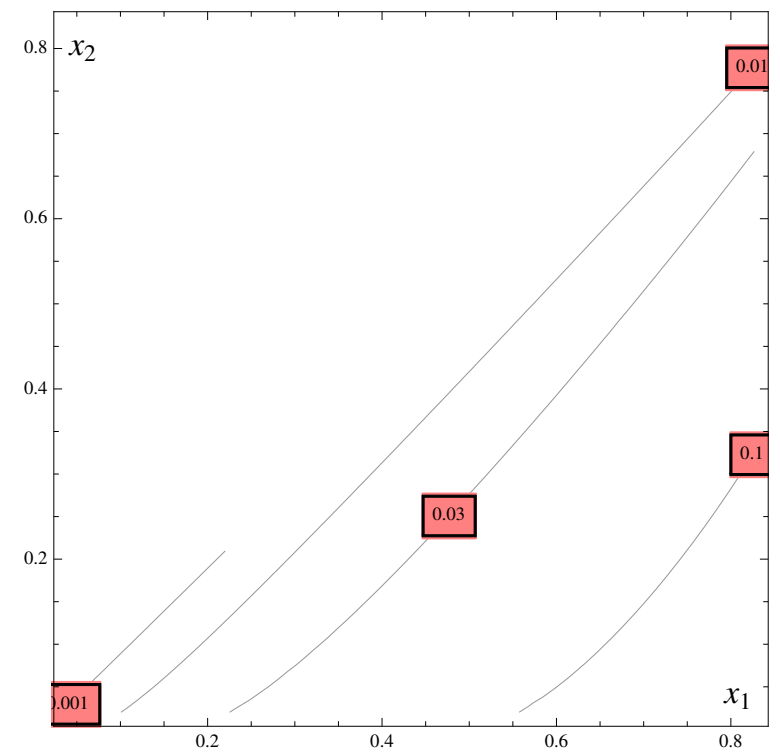

Figure 5. Contour plots of $\lambda_{h N}(0)$ (solid lines) in the maximal scenario, setting $\mu=10 \mathrm{TeV}$, $M=200 \mathrm{GeV}$ and either $y_{L}=1$ or $y_{R}=1$; again, the variables in this plot are $1 / x_{i}$ not $x_{i}$.

Photon-mediated scattering becomes important for lighter mediators. Since our DM is a Majorana fermion, the leading order operator for DM-nucleon coupling is the dimensionsix anapole operator [54]:

$$
\mathcal{O}_{A}=\mathcal{A} \bar{N} \gamma^{\mu} \gamma^{5} N \partial^{\nu} F_{\mu \nu}
$$

The $\mathcal{A}$ can be obtained by integrating out loopy particles step by step [39] or via direct calculation of the loops [55]:

$$
\mathcal{A} \approx-\frac{e\left(\left|\lambda_{i a}^{L}\right|^{2}+\left|\lambda_{i a}^{R}\right|^{2}\right)}{192 \pi^{2} M^{2}}\left(-3 \log \left(x_{i} \epsilon_{a}\right)-\frac{x_{i}+3}{1-x_{i}} \log \frac{x_{i}^{-1}-1}{\sqrt{\epsilon_{a}}}\right),
$$

with $\epsilon_{a}=m_{\ell_{a}}^{2} / M^{2}$. The expression is valid for the heavy leptons with $m_{\mu, \tau}^{2} \gg|Q|^{2}$. It is seen that $\mathcal{A}$ is insensitive to the $\mu$-term and the mixing angle. For $M=100 \mathrm{GeV}$, it is estimated that $\mathcal{A} /\left(\left|\lambda_{i a}^{L}\right|^{2}+\left|\lambda_{i a}^{R}\right|^{2}\right) \sim \mathcal{O}\left(10^{-7}\right) \mathrm{GeV}^{-2}$. The resulting scattering rate is at least four orders of magnitude weaker than the current LUX sensitivity [55].

\section{Conclusion}

In SM, lepton flavor is accidentally conserved but on the other hand LFV is an established fact. So it is of importance to search for LFV processes such as LFV Higgs decay in the LHC era. It is a good probe to new physics. But LFV Higgs decay is negligible and undetectable in most new physics models for addressing neutrino masses. In this paper we study a type of new physics that could lead to large Higgs LFV decay, i.e., lepton-flavored dark matter specified by the particle property of DM (a Majorana fermion) and DM-SM mediators (scalar leptons). Different than other similar setups, here we introduce both the 
left-handed and the right-handed scalar leptons. They allow for large LFV in Higgs decay and thus may explain the tentative $\operatorname{Br}(h \rightarrow \tau \mu) \sim 1 \%$. In particular, we find that the stringent bound from $\tau \rightarrow \mu \gamma$ can be naturally avoided especially in the decoupling limit of slepton sector. Aspects of relic density and radiative direct detection of the leptonic DM are also investigated.

There are several open questions that deserve future investigation. First, as mentioned in the text, neutrino masses and mixings can be radiatively generated because all the core of the Ernest Ma's model is already incorporated in our model. Even restricted to one RHN, i.e., the Majorana DM, we are able to generate realistic neutrino mixings after introducing a couple of scalar lepton doublets $\phi_{l, i}$. Second, in this article we merely discuss LFV in the first and second family of leptons, and such kind of discussions are easily generalized to other families, which is of particular interest when correlated with neutrino phenomenologies. However, it is not easy to reconcile the tiny neutrino mass scale with a large LFV Higgs decay like $\operatorname{Br}(h \rightarrow \tau \mu) \sim 1 \%$, because the former basically requires somewhat smaller Yukawa couplings $\mathcal{O}(0.01)$. Of course, if we work on very light DM like below the $\mathrm{GeV}$ even $\mathrm{MeV}$ scale, maybe there still stands a chance.

\section{Acknowledgments}

We thank Pyungwon Ko very much for valuable discussions and reading the manuscript carefully. This work is supported in part by National Research Foundation of Korea (NRF) Research Grant NRF-2015R1A2A1A05001869 (SB).

\section{A Two- and three-points scalar functions and their limits}

In this appendix we present the technical details used in this paper. The scalar three point function is defined as $[42,56]$.

$$
\begin{aligned}
C_{0}\left(p_{1}, p_{2}, m_{0}, m_{1}, m_{2}\right) & =\frac{(2 \pi \mu)^{4-d}}{i \pi^{2}} \int d^{d} k \frac{1}{\left(k^{2}-m_{0}^{2}\right)\left(\left(k+p_{1}\right)^{2}-m_{1}^{2}\right)\left(\left(k+p_{2}\right)^{2}-m_{2}^{2}\right)} \\
& =-\int_{0}^{1} d x \int_{0}^{x} d y \frac{1}{a x^{2}+b y^{2}+c x y+d x+e y+f-i \epsilon}
\end{aligned}
$$

with

$$
\begin{array}{lll}
a=\left(p_{2}-p_{1}\right)^{2}, & b=p_{1}^{2}, & c=p_{2}^{2}-p_{1}^{2}-\left(p_{2}-p_{1}\right)^{2}, \\
d=m_{1}^{2}-m_{2}^{2}-\left(p_{2}-p_{1}\right)^{2}, & e=m_{0}^{2}-m_{1}^{2}+\left(p_{2}-p_{1}\right)^{2}-p_{2}^{2}, & f=m_{2}^{2} .
\end{array}
$$

When $p_{1}^{2}=p_{2}^{2}$, obviously we have $C_{0}\left(p_{1}, p_{2}, m_{0}, m_{1}, m_{2}\right)=C_{0}\left(p_{1}, p_{2}, m_{0}, m_{2}, m_{1}\right)$. If the invariant masses of the external momentums $p_{1,2}^{2},\left(p_{2}-p_{1}\right)^{2}$ are far lighter than the mass scales of the particles in the loop, $m_{0,1,2}^{2}$, one can approximate $C_{0}\left(p_{1}, p_{2}, m_{0}, m_{1}, m_{2}\right)$ to be

$$
C_{0}\left(m_{0}, m_{1}, m_{2}\right)=-\frac{1}{m_{0}^{2}} G\left(r_{1}, r_{2}\right) \quad \text { with } \quad G\left(r_{1}, r_{2}\right) \equiv \frac{1}{r_{1}-r_{2}}\left(\frac{r_{1} \log r_{1}}{r_{1}-1}-\frac{r_{2} \log r_{2}}{r_{2}-1}\right),
$$



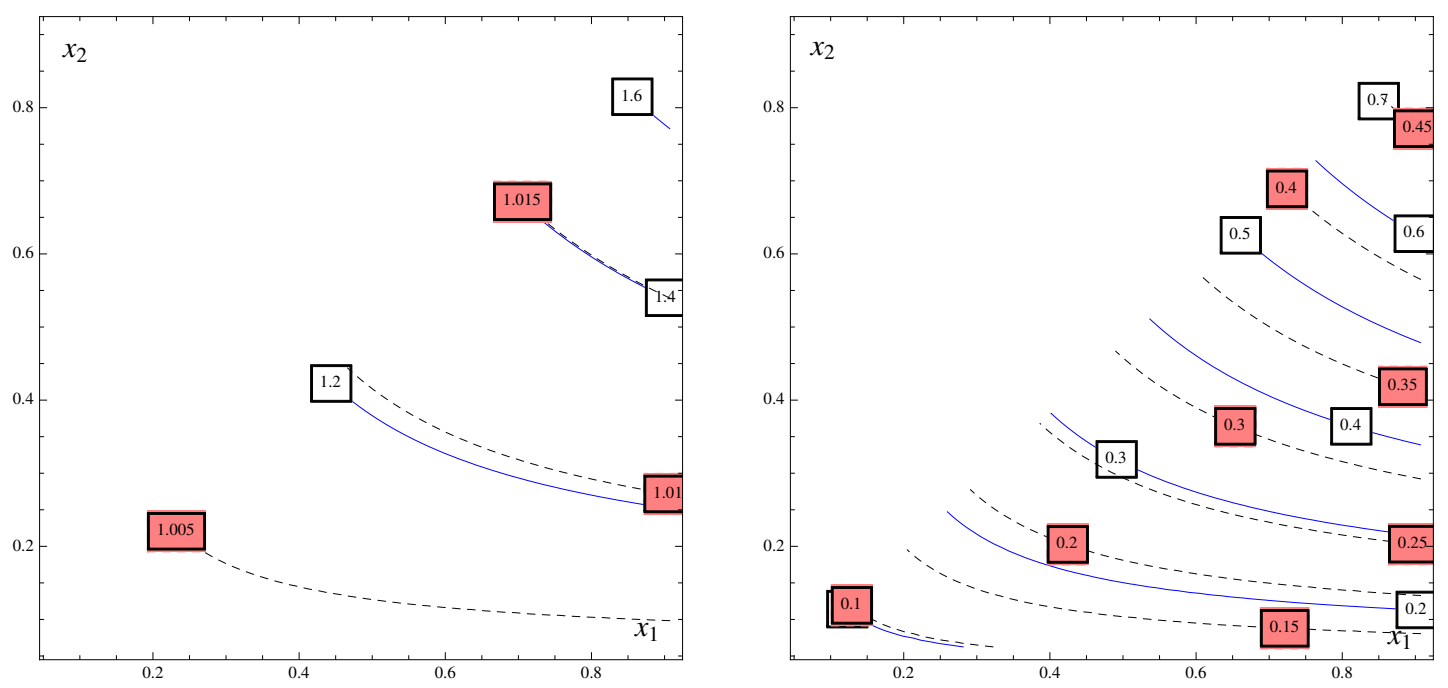

Figure 6. Left: test of approximation; right: distributions of loop integrals. In both panels the dashed lines are for $\left(p_{1}-p_{2}\right)^{2}=0.25 m_{0}^{2}$ and solid lines for $\left(p_{1}-p_{2}\right)^{2}=4 m_{0}^{2} ; x_{i}=m_{0}^{2} / m_{i}^{2}$.

with $r_{i} \equiv m_{i}^{2} / m_{0}^{2}$. Note that $G\left(r_{1}, r_{2}\right)$ is symmetric under interchanging $r_{1}$ and $r_{2}$. There are two particular limits that are helpful in analyzing the radiative decays of Higgs boson.

$\boldsymbol{r}_{\mathbf{2}}=\boldsymbol{r}_{\mathbf{1}}$. For this single propagator case one has

$$
C_{0}\left(m_{0}, m_{1}, m_{1}\right)=-\frac{1}{m_{0}^{2}} G\left(r_{1}\right) \equiv-\frac{1}{m_{0}^{2}} \frac{r_{1}-1-\log r_{1}}{\left(r_{1}-1\right)^{2}} .
$$

If further $r_{1}$ goes to 1 , it slides to $1 / 2 m_{0}^{2}$. But for very heavy $m_{1}$ it decouples as $1 / m_{1}^{2}$.

$r_{2} \gg r_{1} \rightarrow 1$. For the asymmetric propagators like this, we have the simple approximation

$$
C_{0}\left(m_{0}, m_{1}, m_{2}\right) \doteq-\frac{1}{m_{0}^{2}} \frac{1}{\left(r_{2}-1\right)^{2}}\left(1-r_{2}+r_{2} \log r_{2}\right) \approx-\frac{1}{m_{2}^{2}}\left(\log r_{2}-1\right),
$$

Due to the logarithmic factor, it decouples slower than the previous case.

The scalar two-point function is defined as

$$
B_{0}\left(p_{1}, m_{0}, m_{1}\right)=\int d^{d} k \frac{1}{\left(k^{2}-m_{0}^{2}\right)\left(\left(k-p_{1}\right)^{2}-m_{1}^{2}\right)},
$$

which satisfies the relations $B_{0}\left(p_{1}, m_{0}, m_{1}\right)=B_{0}\left(p_{1}, m_{1}, m_{0}\right)$ and $B_{0}\left(p_{1}, m_{0}, m_{1}\right)=$ $B_{0}\left(p_{2}, m_{0}, m_{1}\right)$ for $p_{1}^{2}=p_{2}^{2}$. Actually, it has an explicit expression (up to $\mathcal{O}(\epsilon)$ )

$$
B_{0}\left(p_{1}, m_{0}, m_{1}\right)=\Delta+2-\log \frac{m_{0} m_{1}}{\mu^{2}}+\frac{m_{0}^{2}-m_{1}^{2}}{p_{1}^{2}} \log \frac{m_{1}}{m_{0}}-\frac{m_{0} m_{1}}{p_{1}^{2}}\left(\frac{1}{r}-r\right) \log r,
$$

where $r$ and $1 / r$ are determined by

$$
x^{2}+\frac{m_{0}^{2}+m_{1}^{2}-p_{1}^{2}-i \epsilon}{m_{0} m_{1}} x+1=(x+r)(x+1 / r) .
$$


It has two limits of interest in this paper. Let us consider the first limit, i.e., small external momentum $p_{1}^{2}=Q^{2} \rightarrow 0$, then we have

$$
\begin{aligned}
B_{0}\left(Q, m_{1}, m_{2}\right) \doteq & \frac{x_{1}+x_{2}}{2\left(x_{1}-x_{2}\right)} \log \frac{x_{2}}{x_{1}}+\frac{1}{2} \log \left(x_{1} x_{2}\right)-\left(\log M^{2}+1\right) \\
& +\frac{Q^{2}}{M^{2}} \frac{x_{1} x_{2}\left(x_{1}^{2}-x_{2}^{2}+2 x_{1} x_{2} \log \frac{x_{2}}{x_{1}}\right)}{2\left(x_{1}-x_{2}\right)^{3}}
\end{aligned}
$$

up to irrelevant additive constants that will be cancelled in the expressions. Here $x_{i} \equiv$ $M^{2} / m_{i}^{2}$ with $M$ a referred scale. If $x_{1}=x_{2} \equiv x$, one can greatly simplify it into

$$
B_{0}\left(Q, m_{1}, m_{1}\right) \doteq \log x-\left(2+\log M^{2}\right)+\frac{Q^{2}}{M^{2}} \frac{x}{6} .
$$

Now we move to the other limit, i.e., when one particle in the loop is extraordinarily lighter than other mass scales; without loss of generality, let $m_{1}^{2} \ll m_{2}^{2}, p_{2}^{2}$. Then one has $\left(x_{2} \equiv p_{2}^{2} / m_{2}^{2}\right)$

$$
B_{0}\left(p_{2}, m_{1}, m_{2}\right) \doteq \frac{1}{x_{2}} \log \left(1-x_{2}\right)-2 \operatorname{arctanh}\left(1-2 x_{2}\right)+\frac{1}{2} \log p_{2}^{2} .
$$

\section{B Radiative corrections on Higgs-DM-DM vertex}

In this appendix we derive the approximations of Higgs-DM-DM vertex relevant to DM direct detection. The amplitude is given by $\mathcal{M}+\mathcal{M}_{c}$ with

$$
\mathcal{M} \approx-\bar{u}\left(p_{1}-p_{2}\right) A_{i j}\left(\lambda_{j a}^{L} P_{L}+\lambda_{j a}^{R} P_{R}\right) \gamma^{\mu} C_{\mu}\left(-p_{2}, p_{1}-p_{2}\right)_{a i j}\left(\lambda_{i a}^{L *} P_{R}+\lambda_{i a}^{R *} P_{L}\right) v\left(p_{2}\right),
$$

where terms suppressed by lepton masses are neglected. For short, we denote $C_{\mu}\left(-p_{2}, p_{1}-\right.$ $\left.p_{2}, m_{l_{a}}, m_{\widetilde{e}_{i}}, m_{\widetilde{e}_{j}}\right) \equiv C_{\mu}\left(-p_{2}, p_{1}-p_{2}\right)_{a i j}$. Similar conventions are adopted throughout this paper. It does not cause confusion since we have specified an unique index type for each flavor. The vectorial three-point function can be decomposed into

$$
\gamma^{\mu} C_{\mu}\left(-p_{2}, p_{1}-p_{2}\right)_{a i j}=-\not p_{2} C_{11}\left(-p_{2}, p_{1}-p_{2}\right)_{a i j}+\left(\not p_{1}-\not p_{2}\right) C_{12}\left(-p_{2}, p_{1}-p_{2}\right)_{a i j} .
$$

After using the motion of equation, one has $\not p_{2} \rightarrow-M$ and $\not p_{1}-\not p_{2} \rightarrow+M$. Then, the amplitude takes the form of $\mathcal{M} \approx-\bar{u}\left(p_{1}-p_{2}\right)\left(H_{L} P_{L}+H_{R} P_{R}\right) v\left(p_{2}\right)$ with

$$
H_{L}=\frac{1}{16 \pi^{2}} M A_{i j}\left[\lambda_{i a}^{L *} \lambda_{j a}^{L} C_{11}\left(-p_{2}, p_{1}-p_{2}\right)_{a i j}+\lambda_{i a}^{R *} \lambda_{j a}^{R} C_{12}\left(-p_{2}, p_{1}-p_{2}\right)_{a i j}\right] .
$$

$H_{R}$ is obtained by exchanging $C_{11}$ and $C_{12}$ in $H_{L}$. Specific to the kinematics in this paper, i.e., $p_{2}^{2}=\left(p_{1}-p_{2}\right)^{2}=M^{2}$, and using the equations below eq. (A.6) and eq. (A.2) one can explicitly show $H_{L}=H_{R}$. After some exercise one finds the crossed diagram 
gives $\mathcal{M}_{c}=\mathcal{M}$. Therefore, eventually the form factor relevant to direct detection is $\lambda_{h}(0) \equiv 2\left(H_{L}+H_{R}\right)$. In the $\theta \rightarrow 0$ limit, the leading order is

$$
\begin{aligned}
\lambda_{h N}(0) \approx \sin \theta \frac{\left|y_{L a}\right|^{2}+\left|y_{R a}\right|^{2}}{32 \pi^{2}} \frac{\mu}{\sqrt{2} M}\left[B_{0}\left(p_{1}-p_{2}\right)_{a 1}-B_{0}\left(p_{1}-p_{2}\right)_{a 2}-2 B_{0}\left(p_{1}\right)_{11}\right. \\
+2 B_{0}\left(p_{1}\right)_{12}-C_{0}\left(-p_{2}, p_{1}-p_{2}\right)_{a 12}\left(m_{\widetilde{e}_{1}}^{2}+m_{\widetilde{e}_{2}}^{2}-2 M^{2}\right) \\
\left.+2 C_{0}\left(-p_{2}, p_{1}-p_{2}\right)_{a 11}\left(m_{\widetilde{e}_{1}}^{2}-M^{2}\right)\right] .
\end{aligned}
$$

Note that both the quartic and logarithmic divergencies contained in the two-point functions are cancelled. This is consistent with expectancy and provides as a check for our calculations. It is convenient to write $C_{0}\left(-p_{2}, p_{1}-p_{2}\right)_{a 12}=\mathcal{G}\left(x_{1}, x_{2}\right) / M^{2}$ with

$$
\mathcal{G}\left(x_{1}, x_{2}\right) \approx-\int_{0}^{1} d x \int_{0}^{x} d y \frac{1}{y^{2}-\left(x_{1}+1\right) y+\left(x_{1}-x_{2}\right) x+x_{2}},
$$

with $x_{i}=m_{\widetilde{e}_{i}}^{2} / M^{2}$. It, again, is in the approximation $p_{1}^{2} \rightarrow 0$ and $m_{l_{a}}^{2} \rightarrow 0 ;$ It has an explicit but not illustrative expression, thus not given here.

Open Access. This article is distributed under the terms of the Creative Commons Attribution License (CC-BY 4.0), which permits any use, distribution and reproduction in any medium, provided the original author(s) and source are credited.

\section{References}

[1] R.H. Bernstein and P.S. Cooper, Charged lepton flavor violation: an experimenter's guide, Phys. Rept. 532 (2013) 27 [arXiv:1307.5787] [INSPIRE].

[2] J.D. Bjorken and S. Weinberg, A mechanism for nonconservation of muon number, Phys. Rev. Lett. 38 (1977) 622 [INSPIRE].

[3] R. Harnik, J. Kopp and J. Zupan, Flavor violating Higgs decays, JHEP 03 (2013) 026 [arXiv: 1209.1397] [INSPIRE].

[4] CMS collaboration, Search for Lepton-Flavour-Violating Decays of the Higgs Boson, Phys. Lett. B 749 (2015) 337 [arXiv:1502.07400] [INSPIRE].

[5] ATLAS collaboration, Search for lepton-flavour-violating $H \rightarrow \mu \tau$ decays of the Higgs boson with the ATLAS detector, JHEP 11 (2015) 211 [arXiv:1508.03372] [INSPIRE].

[6] J. Kopp and M. Nardecchia, Flavor and CP-violation in Higgs decays, JHEP 10 (2014) 156 [arXiv: 1406.5303] [INSPIRE].

[7] A. Pilaftsis, Lepton flavor nonconservation in $H^{0}$ decays, Phys. Lett. B 285 (1992) 68 [INSPIRE].

[8] E. Arganda, A.M. Curiel, M.J. Herrero and D. Temes, Lepton flavor violating Higgs boson decays from massive seesaw neutrinos, Phys. Rev. D 71 (2005) 035011 [hep-ph/0407302] [INSPIRE].

[9] E. Arganda, M.J. Herrero, X. Marcano and C. Weiland, Imprints of massive inverse seesaw model neutrinos in lepton flavor violating Higgs boson decays, Phys. Rev. D 91 (2015) 015001 [arXiv: 1405.4300] [INSPIRE]. 
[10] E. Arganda, M.J. Herrero, X. Marcano and C. Weiland, Enhancement of the lepton flavor violating Higgs boson decay rates from SUSY loops in the inverse seesaw model, Phys. Rev. D 93 (2016) 055010 [arXiv: 1508.04623] [INSPIRE].

[11] A. Zee, A theory of lepton number violation, neutrino Majorana mass and oscillation, Phys. Lett. B 93 (1980) 389 [Erratum ibid. B 95 (1980) 461] [INSPIRE].

[12] A. Zee, Quantum numbers of majorana neutrino masses, Nucl. Phys. B 264 (1986) 99 [INSPIRE].

[13] K.S. Babu, Model of 'calculable' Majorana neutrino masses, Phys. Lett. B 203 (1988) 132 [INSPIRE].

[14] E. Ma, Verifiable radiative seesaw mechanism of neutrino mass and dark matter, Phys. Rev. D 73 (2006) 077301 [hep-ph/0601225] [INSPIRE].

[15] D. Aristizabal Sierra and A. Vicente, Explaining the CMS Higgs flavor violating decay excess, Phys. Rev. D 90 (2014) 115004 [arXiv:1409.7690] [InSPIRE].

[16] J. Heeck, M. Holthausen, W. Rodejohann and Y. Shimizu, Higgs $\rightarrow \mu \tau$ in abelian and non-abelian flavor symmetry models, Nucl. Phys. B 896 (2015) 281 [arXiv:1412.3671] [INSPIRE].

[17] Y.-n. Mao and S.-h. Zhu, Higgs boson- $\mu-\tau$ coupling at high and low energy colliders, Phys. Rev. D 93 (2016) 035014 [arXiv:1505.07668] [InSPIRE].

[18] Y. Omura, E. Senaha and K. Tobe, Lepton-flavor-violating Higgs decay $h \rightarrow \mu \tau$ and muon anomalous magnetic moment in a general two Higgs doublet model, JHEP 05 (2015) 028 [arXiv: 1502.07824] [INSPIRE].

[19] I. Doršner et al., New physics models facing lepton flavor violating Higgs decays at the percent level, JHEP 06 (2015) 108 [arXiv:1502.07784] [INSPIRE].

[20] A. Crivellin, G. D'Ambrosio and J. Heeck, Explaining $h \rightarrow \mu^{ \pm} \tau^{\mp}, B \rightarrow K^{*} \mu^{+} \mu^{-}$and $B \rightarrow K \mu^{+} \mu^{-} / B \rightarrow K e^{+} e^{-}$in a two-Higgs-doublet model with gauged $L_{\mu}-L_{\tau}$, Phys. Rev. Lett. 114 (2015) 151801 [arXiv:1501.00993] [INSPIRE].

[21] F.J. Botella, G.C. Branco, M. Nebot and M.N. Rebelo, Flavour changing Higgs couplings in a class of two Higgs doublet models, arXiv:1508.05101 [INSPIRE].

[22] M.D. Campos, A.E.C. Hernández, H. Päs and E. Schumacher, Higgs $\rightarrow \mu \tau$ as an indication for $S_{4}$ flavor symmetry, Phys. Rev. D 91 (2015) 116011 [arXiv:1408.1652] [INSPIRE].

[23] L. de Lima, C.S. Machado, R.D. Matheus and L.A.F. do Prado, Higgs flavor violation as a signal to discriminate models, JHEP 11 (2015) 074 [arXiv:1501.06923] [INSPIRE].

[24] X. Liu, L. Bian, X.-Q. Li and J. Shu, $h \rightarrow \mu \tau$, muon $g-2$ and a possible interpretation of the Galactic Center $\gamma$ ray excess, arXiv:1508.05716 [INSPIRE].

[25] A. Crivellin, J. Heeck and P. Stoffer, A perturbed lepton-specific two-Higgs-doublet model facing experimental hints for physics beyond the standard model, Phys. Rev. Lett. 116 (2016) 081801 [arXiv: 1507.07567] [INSPIRE].

[26] C.-W. Chiang, H. Fukuda, M. Takeuchi and T.T. Yanagida, Flavor-changing neutral-current decays in top-specific variant axion model, JHEP 11 (2015) 057 [arXiv:1507.04354] [INSPIRE].

[27] W. Huang and Y.-L. Tang, Flavor anomalies at the LHC and the R-parity violating supersymmetric model extended with vectorlike particles, Phys. Rev. D 92 (2015) 094015 [arXiv: 1509.08599] [INSPIRE].

[28] X.-G. He, J. Tandean and Y.-J. Zheng, Higgs decay $h \rightarrow \mu \tau$ with minimal flavor violation, JHEP 09 (2015) 093 [arXiv: 1507.02673] [INSPIRE]. 
[29] W. Altmannshofer, S. Gori, A.L. Kagan, L. Silvestrini and J. Zupan, Uncovering mass generation through Higgs flavor violation, Phys. Rev. D 93 (2016) 031301 [arXiv: 1507.07927] [INSPIRE].

[30] K. Cheung, W.-Y. Keung and P.-Y. Tseng, Leptoquark induced rare decay amplitudes $h \rightarrow \tau^{\mp} \mu^{ \pm}$and $\tau \rightarrow \mu \gamma$, Phys. Rev. D 93 (2016) 015010 [arXiv: 1508.01897] [InSPIRE].

[31] S. Baek and K. Nishiwaki, Leptoquark explanation of $h \rightarrow \mu \tau$ and muon $(g-2)$, Phys. Rev. D 93 (2016) 015002 [arXiv: 1509.07410] [INSPIRE].

[32] X.-J. Bi, P.-H. Gu, T. Li and X. Zhang, ATIC and PAMELA results on cosmic $e^{ \pm}$excesses and neutrino masses, JHEP 04 (2009) 103 [arXiv:0901.0176] [INSPIRE].

[33] C.-J. Lee and J. Tandean, Lepton-flavored scalar dark matter with minimal flavor violation, JHEP 04 (2015) 174 [arXiv: 1410.6803] [INSPIRE].

[34] P. Agrawal, Z. Chacko, C. Kilic and C.B. Verhaaren, A couplet from flavored dark matter, JHEP 08 (2015) 072 [arXiv: 1503.03057] [InSPIRE].

[35] A. Hamze, C. Kilic, J. Koeller, C. Trendafilova and J.-H. Yu, Lepton-flavored asymmetric dark matter and interference in direct detection, Phys. Rev. D 91 (2015) 035009 [arXiv:1410.3030] [INSPIRE].

[36] Y. Bai and J. Berger, Lepton portal dark matter, JHEP 08 (2014) 153 [arXiv:1402.6696] [INSPIRE].

[37] C.-Q. Geng, D. Huang and L.-H. Tsai, X-ray line from the dark transition electric dipole, JHEP 08 (2014) 086 [arXiv: 1406.6481] [INSPIRE].

[38] J. Kile, A. Kobach and A. Soni, Lepton-flavored dark matter, Phys. Lett. B 744 (2015) 330 [arXiv: 1411.1407] [INSPIRE].

[39] P. Agrawal, S. Blanchet, Z. Chacko and C. Kilic, Flavored dark matter and its implications for direct detection and colliders, Phys. Rev. D 86 (2012) 055002 [arXiv:1109.3516] [INSPIRE].

[40] S. Chang, R. Edezhath, J. Hutchinson and M. Luty, Leptophilic effective WIMPs, Phys. Rev. D 90 (2014) 015011 [arXiv: 1402.7358] [INSPIRE].

[41] LUX collaboration, D.S. Akerib et al., First results from the LUX dark matter experiment at the Sanford Underground Research Facility, Phys. Rev. Lett. 112 (2014) 091303 [arXiv: 1310.8214] [INSPIRE].

[42] A. Denner, Techniques for calculation of electroweak radiative corrections at the one loop level and results for W physics at LEP-200, Fortsch. Phys. 41 (1993) 307 [arXiv:0709.1075] [INSPIRE].

[43] BABAR collaboration, B. Aubert et al., Searches for lepton flavor violation in the decays $\tau^{ \pm} \rightarrow e^{ \pm} \gamma$ and $\tau^{ \pm} \rightarrow \mu^{ \pm} \gamma$, Phys. Rev. Lett. 104 (2010) 021802 [arXiv:0908.2381] [INSPIRE].

[44] T. Aushev et al., Physics at super B factory, arXiv:1002.5012 [INSPIRE].

[45] MEG collaboration, J. Adam et al., New constraint on the existence of the $\mu^{+} \rightarrow e^{+} \gamma$ decay, Phys. Rev. Lett. 110 (2013) 201801 [arXiv:1303.0754] [InSPIRE].

[46] D. Carmi, A. Falkowski, E. Kuflik, T. Volansky and J. Zupan, Higgs after the discovery: a status report, JHEP 10 (2012) 196 [arXiv:1207.1718] [INSPIRE].

[47] J. Guo, Z. Kang, J. Li and T. Li, Implications of Higgs sterility for the Higgs and stop sectors, arXiv:1308.3075 [inSPIRE].

[48] J. Bernon, B. Dumont and S. Kraml, Status of Higgs couplings after Run 1 of the LHC, Phys. Rev. D 90 (2014) 071301 [arXiv: 1409.1588] [INSPIRE]. 
[49] M. Garny, A. Ibarra and S. Vogl, Signatures of Majorana dark matter with t-channel mediators, Int. J. Mod. Phys. D 24 (2015) 1530019 [arXiv: 1503.01500] [INSPIRE].

[50] K. Griest and D. Seckel, Three exceptions in the calculation of relic abundances, Phys. Rev. D 43 (1991) 3191 [INSPIRE].

[51] X. Gao, Z. Kang and T. Li, Origins of the isospin violation of dark matter interactions, JCAP 01 (2013) 021 [arXiv:1107.3529] [INSPIRE].

[52] J.M. Alarcon, J. Martin Camalich and J.A. Oller, The chiral representation of the $\pi N$ scattering amplitude and the pion-nucleon sigma term, Phys. Rev. D 85 (2012) 051503 [arXiv:1110.3797] [INSPIRE].

[53] A. Crivellin, M. Hoferichter and M. Procura, Accurate evaluation of hadronic uncertainties in spin-independent WIMP-nucleon scattering: disentangling two- and three-flavor effects, Phys. Rev. D 89 (2014) 054021 [arXiv:1312.4951] [INSPIRE].

[54] B. Kayser and A.S. Goldhaber, CPT and CP properties of Majorana particles and the consequences, Phys. Rev. D 28 (1983) 2341 [INSPIRE].

[55] J. Kopp, L. Michaels and J. Smirnov, Loopy constraints on leptophilic dark matter and internal bremsstrahlung, JCAP 04 (2014) 022 [arXiv: 1401.6457] [INSPIRE].

[56] G. 't Hooft and M. Veltman, Scalar one loop integrals, Nucl. Phys. 153 (1979) 365 [InSPIRE] 